\title{
NOTES ON THE GEOMETRY OF SPACE OF POLYNOMIALS
}

\author{
HAN JU LEE
}

\begin{abstract}
We show that the symmetric injective tensor product space $\hat{\otimes}_{n, s, \varepsilon} E$ is not complex strictly convex if $E$ is a complex Banach space of $\operatorname{dim} E \geq 2$ and if $n \geq 2$ holds. It is also reproved that $\ell_{\infty}$ is finitely represented in $\hat{\otimes}_{n, s, \varepsilon} E$ if $E$ is infinite dimensional and if $n \geq 2$ holds, which was proved in the other way in [3].
\end{abstract}

The real geometric properties of spaces of polynomials are discussed in [1, 6. In particular, it is shown that the symmetric injective tensor product space $\hat{\otimes}_{n, s, \varepsilon} E$ is not strictly convex if $E$ is a Banach space of $\operatorname{dim} E \geq 2$ and if $n \geq 2$ holds.

Let $E$ be a Banach space over a real or complex filed and $E^{\prime}$ is denoted as the Banach dual of $E$. An element $x$ in the unit sphere $S_{E}$ is called a (real) extreme point of the unit ball $B_{E}$ if for some $y \in E$,

$$
\|x \pm y\| \leq 1
$$

implies $y=0$. Recall that a Banach space $E$ is said to be strictly convex if every element of $S_{X}$ is an extreme point of $B_{E}$. Suppose for the moment that $E$ is a complex Banach space. An element $x$ in the unit sphere $S_{E}$ is said to be a complex extreme point if for some $y \in E$,

$$
\sup \{\|x+\zeta y\|: \zeta \in \mathbf{C},|\zeta|=1\} \leq 1
$$

implies $y=0$. A complex Banach space $E$ is said to be complex strictly convex if every point in $S_{E}$ is a complex extreme point of $B_{E}$. Notice that if a complex Banach space is not complex strictly convex, then it is not strictly convex.

Given a Banach space $E$, the space $\otimes_{n, s} E$ consists of all tensors of the form

$$
u=\sum_{j=1}^{k} \lambda_{j} x_{j} \otimes x_{j} \otimes x_{j} \cdots \otimes x_{j},
$$

where $x_{j}$ are elements in $E$ for $j=1, \ldots, k$ and $\lambda_{j}$ 's are scalars. Now define its injective norm as

$$
\|u\|_{\varepsilon}=\sup \left\{\left|\sum_{j=1}^{k} \lambda_{j}\left\langle\phi, x_{j}\right\rangle^{n}\right|: \phi \in B_{E^{\prime}}\right\} .
$$

We denote the completion of $\otimes_{n, s} E$ with this norm by $\hat{\otimes}_{n, s, \varepsilon} E$. It is clear that $\hat{\otimes}_{n, s, \varepsilon} E^{\prime}$ is a closed subspace of space $\mathcal{P}\left({ }^{n} E\right)$ of all $n$-homogeneous polynomials (For more details, consult [2, 4.)

2000 Mathematics Subject Classification. 46B20.

Key words and phrases. Complex strictly convex, Polynomials, Symmetric injective tensor product, Finite representability.

The author acknowledges the financial support of the Korean Research Foundation made in the program year of 2002 (KRF-2002-070-C00005). 


\section{Main Results}

We begin with the following useful observation which is a modification of Theorem 2.2 in [7].

Lemma 1.1. Let $F$ be a finite $m$-dimensional Banach space with the Banach-Mazur distance $d\left(F, \ell_{2}^{m}\right) \leq d$. Then there exist $\left\{x_{j}\right\}_{j=1}^{m}$ in $F$ and $\left\{\phi_{j}\right\}_{j=1}^{m}$ in $S_{F^{\prime}}$ such that for each $i=1, \ldots, m$,

$$
\begin{gathered}
1 \leq\left\langle\phi_{i}, x_{i}\right\rangle \leq d, \quad\left\langle\phi_{j}, x_{k}\right\rangle=0 \quad \text { for all different } j, k \\
1 \leq \min _{1 \leq k \leq m}\left\|x_{k}\right\| \leq \max _{1 \leq k \leq m}\left\|x_{k}\right\|=\left\|x_{1}\right\| \leq d
\end{gathered}
$$

and for every real $r \geq 2$,

$$
\sup \left\{\left(\sum_{k=1}^{m}\left|\left\langle\phi, x_{k}\right\rangle\right|^{r}\right)^{\frac{1}{r}}: \phi \in B_{F^{\prime}}\right\}=\left\|x_{1}\right\|
$$

Proof. We shall identify $\left(\ell_{2}^{m}\right)^{\prime}$ with $\ell_{2}^{m}$. Consider an isomorphism $T: \ell_{2}^{m} \rightarrow F$ with $\|T\| \leq d$ and $\left\|T^{-1}\right\|=1$. Since $\left\|\left(T^{*}\right)^{-1}\right\|=1$, we get $\|\phi\| \leq\left\|T^{*}(\phi)\right\|_{2}$ for every $\phi \in F^{\prime}$. Then there exists $\phi_{1} \in S_{F^{\prime}}$ such that

$$
1 \leq\left\|T^{*}\left(\phi_{1}\right)\right\|_{2}=\max \left\{\left\|T^{*}(\phi)\right\|_{2}: \phi \in B_{F^{\prime}}\right\} \leq d .
$$

Then there exists $\phi_{2} \in S_{F^{\prime}}$ such that

$$
\begin{aligned}
1 & \leq\left\|T^{*}\left(\phi_{2}\right)\right\|_{2} \\
& =\max \left\{\left\|T^{*}(\phi)\right\|_{2}: \phi \in B_{F^{\prime}}, \quad\left\langle T^{*}(\phi), \overline{T^{*}\left(\phi_{1}\right)}\right\rangle=\left\langle T^{*}(\phi), T^{*}\left(\phi_{1}\right)\right\rangle_{2}=0\right\},
\end{aligned}
$$

where $\langle\cdot, \cdot\rangle_{2}$ is the standard inner product in $\ell_{2}^{m}$ and $\bar{x}$ denotes $\{\overline{x(k)}\}_{k=1}^{m}$ if $x=$ $\{x(k)\}_{k=1}^{m}$.

In this way, we obtain $m$ vectors $\left\{\phi_{k}\right\}_{k=1}^{m}$ in $S_{F^{\prime}}$ such that $\left\{T^{*}\left(\phi_{k}\right)\right\}_{k=1}^{m}$ are orthogonal. Taking $w_{k}=\overline{T^{*}\left(\phi_{k}\right)} /\left\|T^{*}\left(\phi_{k}\right)\right\|_{2}$ for each $k=1, \ldots, m$, let $x_{k}=T\left(w_{k}\right)$ for each $k=1, \ldots, m$. Notice that

$$
\left\langle\phi_{i}, x_{j}\right\rangle=\left\langle\phi_{i}, T\left(w_{j}\right)\right\rangle=\left\langle T^{*}\left(\phi_{i}\right), w_{j}\right\rangle=\left\|T^{*}\left(\phi_{j}\right)\right\|_{2} \delta_{i j}
$$

where $\delta_{i j}=1$ if $i=j$ and $\delta_{i j}=0$ if $i \neq j$. So (1) is satisfied.

Since $\left\{w_{k}\right\}_{k=1}^{m}$ is an orthonormal basis in $\ell_{2}^{m}$, we get for each $\phi \in B_{F^{\prime}}$

$$
\begin{aligned}
\left(\sum_{k=1}^{m}\left|\left\langle\phi, x_{k}\right\rangle\right|^{2}\right)^{\frac{1}{2}} & =\left(\sum_{k=1}^{m}\left|\left\langle\phi, T\left(w_{k}\right)\right\rangle\right|^{2}\right)^{\frac{1}{2}} \\
& =\left(\sum_{k=1}^{m}\left|\left\langle T^{*}(\phi), w_{k}\right\rangle\right|^{2}\right)^{\frac{1}{2}}=\left\|T^{*}(\phi)\right\|_{2} \leq\left\|T^{*}\left(\phi_{1}\right)\right\|_{2} .
\end{aligned}
$$

Hence we have

$$
\sup \left\{\left(\sum_{k=1}^{m}\left|\left\langle\phi, x_{k}\right\rangle\right|^{r}\right)^{\frac{1}{r}}: \phi \in B_{F^{\prime}}\right\}=\left\|T^{*}\left(\phi_{1}\right)\right\|_{2}=\left\langle\phi_{1}, x_{1}\right\rangle \leq\left\|x_{1}\right\| .
$$


It is also clear that

$$
\sup \left\{\left(\sum_{k=1}^{m}\left|\left\langle\phi, x_{k}\right\rangle\right|^{r}\right)^{\frac{1}{r}}: \phi \in B_{F^{\prime}}\right\} \geq \max _{1 \leq j \leq m}\left\|x_{j}\right\| .
$$

Notice also that $1 \leq\left\|x_{j}\right\|=\left\|T\left(w_{j}\right)\right\| \leq d$ for every $1 \leq j \leq m$. Hence we have obtained $m$ vectors $\left\{x_{1}, \ldots, x_{m}\right\}$ satisfying equations (1.2) and (1.3).

Boyd and Ryan showed in [1] that $\hat{\otimes}_{n, s, \varepsilon} E$ is not real strictly convex for every $n \geq 2$ and $\operatorname{dim} E \geq 2$. In the case of a complex Banach space we have the following

Theorem 1.2. Let $E$ be a real (resp. complex) Banach space of $\operatorname{dim} \geq 2$. Then for $n \geq 2$ the space $\hat{\otimes}_{n, s, \varepsilon} E$ and $\mathcal{P}\left({ }^{n} E\right)$ are not real (resp. complex) strictly convex.

Proof. We shall prove the complex case. The proof can be easily applied to the real case. It is enough to show that $\hat{\otimes}_{n, s, \varepsilon} E$ is not complex strictly convex. For each two dimensional subspace $F$ of $E$, by applying Lemma 1.1, we have two vectors $x_{1}, x_{2}$ such that $1 \leq\left\|x_{2}\right\| \leq\left\|x_{1}\right\|$ and

$$
\sup _{\phi \in B_{F^{\prime}}}\left(\left|\left\langle\phi, x_{1}\right\rangle\right|^{n}+\left|\left\langle\phi, x_{2}\right\rangle\right|^{n}\right)=\left\|x_{1}\right\|^{n} .
$$

Now let

$$
\begin{aligned}
& u=\frac{1}{\left\|x_{1}\right\|^{n}} x_{1} \otimes x_{1} \otimes \cdots \otimes x_{1}, \\
& v=\frac{1}{\left\|x_{1}\right\|^{n}} x_{2} \otimes x_{2} \otimes \cdots \otimes x_{2} .
\end{aligned}
$$

Then $\|u\|_{\varepsilon}=1$ and $v \neq 0$. Notice that for each $|\zeta| \leq 1$,

$$
\|u+\zeta v\|_{\varepsilon}=\frac{1}{\left\|x_{1}\right\|^{n}} \sup _{\phi \in B_{E^{\prime}}}\left|\left\langle\phi, x_{1}\right\rangle^{n}+\zeta\left\langle\phi, x_{2}\right\rangle^{n}\right| \leq 1 .
$$

Therefore $u$ is not a complex extreme point. The proof is done.

In the following theorem we reprove the Dineen's result in 3 by applying Lemma 1.1

Theorem 1.3. [3] Let $E$ be an infinite dimensional Banach space. Then $\ell_{\infty}$ is finitely represented in $\hat{\otimes}_{n, s, \varepsilon} E$ and $\mathcal{P}\left({ }^{n} E\right)$ for every $n \geq 2$.

Proof. By the Dvoretzky theorem [5] we can choose for any positive integer $m$ and any $\epsilon>0$ an $m$ dimensional subspace $F$ in $E$ with $d\left(F, \ell_{2}^{m}\right) \leq 1+\epsilon$. By Lemma 1.1. we have $m$ elements $\left\{x_{j}\right\}$ in $F$ and $\left\{\phi_{j}\right\}$ in $S_{F^{\prime}}$ satisfying (1.1), (1.2) and (1.3) with $d=1+\epsilon$. For any $\left(\alpha_{i}\right)_{i=1}^{m} \in S_{\ell_{\infty}^{m}}$, using the Hahn-Banach extension,

$$
\begin{aligned}
\left\|\sum_{i=1}^{m} \alpha_{i} x_{i} \otimes \cdots \otimes x_{i}\right\|_{\varepsilon} & =\sup _{\phi \in B_{E^{\prime}}}\left|\sum_{i=1}^{m} \alpha_{i}\left\langle\phi, x_{i}\right\rangle^{n}\right| \\
& \geq \max _{1 \leq j \leq m}\left|\alpha_{j}\right|\left\langle\phi_{j}, x_{j}\right\rangle \geq 1,
\end{aligned}
$$

and we also get

$$
\begin{aligned}
\left\|\sum_{i=1}^{m} \alpha_{i} x_{i} \otimes \cdots \otimes x_{i}\right\|_{\varepsilon} & =\sup _{\phi \in B_{E^{\prime}}}\left|\sum_{i=1}^{m} \alpha_{i}\left\langle\phi, x_{i}\right\rangle^{n}\right| \\
& \leq \sup _{\phi \in B_{E^{\prime}}} \sum_{i=1}^{m}\left|\alpha_{i}\right|\left|\left\langle\phi, x_{i}\right\rangle\right|^{n} \leq\left\|x_{1}\right\|^{n} \leq(1+\epsilon)^{n} .
\end{aligned}
$$


The proof is complete.

\section{REFERENCES}

[1] C. Boyd and R.A. Ryan, Geometric theory of spaces of integral polynomials and symmetric tensor products, J. Funct. Anal. 179 (2001), no. 1, 18-42.

[2] S.B. Chae, Holomorphy and calculus in normed spaces, Marcel Dekker, Inc., New York, 1985.

[3] S. Dineen, A Dvoretzky theorem for polynomials, Proc. Amer. Math. Soc. 123 (1995), no. 9, $2817-2821$.

[4] S. Dineen, Complex analysis on infinite-dimensional spaces, Springer-Verlag London, Ltd., London, 1999.

[5] A. Dvoretzky, Some results on convex bodies and Banach spaces, Proc. Internat. Sympos. Linear Spaces (Jerusalem, 1960), Jerusalem Academic Press, 1961, pp. 123-160.

[6] R.A. Ryan and B. Turett, Geometry of spaces of polynomials, J. Math. Anal. Appl. 221 (1998), no. 2, 698-711.

[7] A. Kamińska and H.J. Lee, On uniqueness of extension of homogeneous polynomials, Houston J. Math. 32 (2006), no. 1, 227-252.

Department of Mathematics, POStech, San 31, Hyoja-dong, Nam-gu, Pohang-shi, Kyungbuk, Republic of Korea, +82-054-279-2712

E-mail address: hahnju@postech.ac.kr 